\title{
Nuevas fitocenosis del Carso de Baire, Cuba Oriental
}

\author{
Important phytocenoses of Carso de Baire, Eastern Cuba
}

\author{
Orlando J. Reyes ${ }^{1}$ \& Félix Acosta Cantillo ${ }^{1}$
}

\begin{abstract}
Resumen
El Carso de Baire es una zona constituida fundamentalmente por mogotes y poljas que se encuentran en la vertiente Norte de la Sierra Maestra y se diferencia del resto de la misma por su geología y geomorfología. Generalmente no hay un suelo típico, la temperatura del aire varía entre 20 y $24^{\circ} \mathrm{C}$ y llueve de 1.200 a $1.600 \mathrm{~mm}$ por año. Mediante la utilización de la metodología de la Escuela Zurich-Montpellier, se describen las dos asociaciones más conspicuas y extensas de dicha zona, Hohenbergio penduliflorae - Guapiretum obtusatae y Rhipsali cassuthae - Hohenbergietum penduliflorae, las que se incluyen en la alianza Synapsidi ilicifoliae - Coccothrinacion Borhidi 1996. La primera, constituida por un Bosque siempreverde micrófilo con poca cobertura, ocupa la mayor extensión en el lapiez de la parte alta de los mogotes, desarrollando el reciclaje de nutrientes en la estera radical; a su vez la segunda, que conforma un matorral, crece en los paredones de alrededor de 90 grados y sus plantas se fijan a las fisuras de las rocas. Ambas asociaciones están sometidas a un gran estrés hídrico y nutrimental.
\end{abstract}

Palabras clave: asociaciones vegetales, Carso de Baire, Cuba Oriental, mogotes, sintaxonomía.

\begin{abstract}
Baire's karst is encountered on the north slope of Sierra Maestra. It is geologically and geomorphologically different from the rest of the Sierra Meaestra mountain system because of the development of "mogotes" and "poljas". Generally no soil type is typical of the region, air temperature varies from 20 to $24^{\circ} \mathrm{C}$ and rainfall is 1,200 to $1,600 \mathrm{~mm}$ annually. Using the Zurich-Montpellier school methodology the two most important phytocenoses are described from Carso de Baire Hohenbergio penduliflorae - Guapiretum obtusatae and Rhipsali cassuthae - Hohenbergietum penduliflorae, included in the Synapsidi ilicifoliae - Coccothrinacion Borhidi 1996 alliance. The first is represented by an open, microphyllous, evergreen forest occupying the highest part of "mogotes" (karstic dome hill), whereas the second consists of shrubby vegetation growing on a 90-degree wall. Both occur under water and nutritional stress.
\end{abstract}

Key words: Carso de Baire, karstic dome hill, syntaxonomy, associations, Eastern Cuba.

\section{Introducción}

Cuba Oriental, constituida por los macizos montañosos Sagua Baracoa, Sierra Maestra y las áreas aledañas e intermedias se destaca por un desarrollo paleogeográfico particular (Iturralde Vinent 2007) y sus montañas son muy antiguas, desde el Cretácico superior y el Eoceno respectivamente (Oro 1989; Reyes 2000). A dichas montañas llegaron las principales migraciones de la biodiversidad, evolucionaron y originaron el mayor endemismo del archipiélago cubano (López 1998, 2005).

La zona conocida como Carso de Baire (Núñez \& Viña 1989) se encuentra en la parte Norte de la
Sierra Maestra, diferenciándose del resto de dicha sierra por su geología y geomorfología, ya que tiene mogotes y poljas. Se extiende desde los alrededores de Guisa hasta las inmediaciones de Matías.

En el Carso de Baire fue referida por Borhidi (1991) la asociación Coccothrinaci - Tabebuietum bibracteolatae Borhidi \& Muñiz 1991. Posteriormente se describieron (Reyes \& Acosta 2004b) las asociaciones secundarias Cupanio glabrae - Ocoteetum coriaceae Reyes \& Acosta 2004, Ocoteo coriaceae - Alvaradoetum arborescentis Reyes \& Acosta 2004 y Guareo guidoniae - Dendropanacetum arborei Reyes \&

${ }^{1}$ Centro Oriental de Ecosistemas y Biodiversidad (BIOECO), Santiago de Cuba, Ministerio de Ciencia, Tecnología y Medio Ambiente (CITMA), Cuba. Correo electrónico: joel@bioeco.ciges.inf.cu. 
Acosta 2004 en condiciones edáficas más favorables que las expuestas en este trabajo.

Aunque este territorio tiene gran importancia por su biodiversidad y unicidad fitocenótica, se han desarrollado pocos estudios fitosociológicos sobre esta área. Recientemente Borhidi (1991, 1996) y Reyes \& Acosta (2004a,b) describieron fitocenosis de diversa categoría sobre esta localidad, pero aún incompletos, probablemente debido a la alta diversidad. El objetivo de este trabajo es describir dos nuevas fitocenosis en estos mogotes, con vistas a fundamentar mejor su protección.

\section{Materiales y Métodos}

\section{Descripción del área de estudio}

La zona de estudio está delimitada por las cuadrículas: $\mathrm{X}_{1}=76^{\circ} 26^{\prime} 40,73^{\prime}, \mathrm{Y}_{1}=20^{\circ} 09^{\prime} 55,56^{\prime \prime} \mathrm{X}_{2}=$ $76^{\circ} 20^{\prime} 05,97^{\prime \prime}, Y_{2}=20^{\circ} 13^{\prime} 48,15^{\prime \prime}$. Está constituida fundamentalmente por mogotes y poljas (Núñez et al. 1989); es decir, mesetas denudativas fuertemente carsificadas y diseccionadas (Viña 1991) con paredes verticales. Las rocas son calizas organógeno-detríticas, mármoles y conglomerados polimícticos que se corresponden con la formación eocénica Charco Redondo, las que sobreyacen las rocas más extendidas en la Sierra Maestra conocidas como Grupo El Cobre. Por ello, en la parte alta y paredes de los mogotes generalmente no existe un verdadero suelo, sino las plantas se fijan a las fisuras de las rocas o crecen en una estera radical (conjunto de mantillo, raíces y raicillas) en el lapiez o diente de perro (dogtooth).

La temperatura media anual del aire es de 20 a $22^{\circ} \mathrm{C}$ en las partes altas y de 22 a $24^{\circ} \mathrm{C}$ en las más bajas (Lapinel 1989). La precipitación media anual varía desde alrededor de $1.200 \mathrm{~mm}$ en las áreas menos elevadas hasta algo más de $1.600 \mathrm{~mm}$ en las más altas (Gagua et al. 1989). La lluvia promedio en La Tabla es de 1.634 $\mathrm{mm}$, variando entre los años desde 1.216 hasta 2.320 $\mathrm{mm}$; como promedio hay 94 días con lluvia en el año. Se definen claramente un período lluvioso de marzo a noviembre con el $85 \%$ de las precipitaciones y otro menos lluvioso de diciembre a febrero con el $15 \%$. Las lluvias más frecuentes caen entre 5,1 y $30 \mathrm{~mm}$. La humedad relativa del aire tiene una media anual de 80 a $85 \%$ (Montenegro 1991) y la evaporación media anual es de 1.300 a $1.500 \mathrm{~mm}$.

\section{Metodología}

Las muestras de vegetación (listas) se hicieron por el método de Braun Blanquet (1921, 1951, 1964), utilizando los resultados de varios autores (Samek 1973; Borhidi 1991). Dichas muestras (mínimo seis por asociación) tienen forma rectangular y un área mínima de $400 \mathrm{~m}^{2}$, en ella la abundancia-dominancia de cada especie se estimó en cada estrato con la escala siguiente: $5=$ cuando cubre $75 \%$ o más del área de la muestra; 4 cubre 50 a $75 \% ; 3$ cubre 25 a $50 \% ; 2$ cubre 5 a $25 \%$; 1 cubre menos del 5\%; "+" con pocos ejemplares aislados y con poca cobertura y "r" cuando eran dos o tres ejemplares aislados con poca importancia cenológica. La sociabilidad se anotó para cada especie, separada por un punto del valor de la escala combinada de abundancia-dominancia; los valores tienen los siguientes significados: (1) cada individuo crece aislado; (2) establecen pequeños grupos; (3) forman manchas o cojines; (4) se desarrollan en colonias o manchas grandes; (5) conforman grandes colonias o poblaciones puras. Los estratos considerados fueron (Samek 1973): arbóreo ( $\left.\mathrm{E}_{3}\right)$, con más de $5 \mathrm{~m}$ de altura; arbustivo $\left(\mathrm{E}_{2}\right)$, entre 2 y $5 \mathrm{~m}$; herbáceo $\left(E_{1}\right)$, menos de $2 \mathrm{~m}$. Se consideran subestratos cuando dentro de un estrato hay conjuntos de elementos que tienen alturas bien definidas y que son diferentes entre si.

Además, se realizaron observaciones del ecótopo en el sitio de la muestra y sus alrededores. En el lugar de la lista se midieron o estimaron: la inclinación de la pendiente, la exposición, la altitud, la posición en el mogote, el macrorelieve, así como el micro y nano relieve.

La ordenación de las muestras de vegetación y la separación de las fitocenosis se efectuó por métodos fitocenológicos (Scamoni \& Passarge 1963). Los grados de presencia de las especies se establecieron según la escala siguiente (Greig-Smith 1964): clase I, presentes de 1 a $20 \%$ de las listas; clase II, de 21 a 40\%; clase III, de 41 a 60\%; clase IV, de 61 a $80 \%$ y clase V, de 81 a $100 \%$. Luego de organizada la tabla (y unificadas las fitocenosis), se realizó la comprobación de la homogeneidad de acuerdo a la ley de Raunkiaer (1934), la que expone que en una asociación homogénea las frecuencias de las constancias (o presencias) siguen una curva típica en forma de jota invertida, o sea, la relación de las constancias sigue la fórmula: I $>$ II $>$ III $<=>$ IV $<$ V. Para la combinación característica de la asociación se utilizaron las especies con grados de presencia IV y V; para las subasociaciones se usaron las combinaciones diferenciales (Scamoni \& Passarge 1959).

Para la categorización y la nomenclatura de los rangos principales de las fitocenosis se siguió el Principio II y el artículo 11 del Código de Nomenclatura Fitosociológica (Barkman et al. 1988), los que en orden 
jerárquico creciente son: asociación, alianza, orden y clase; así como el de los rangos auxiliares: subasociación, subalianza, suborden y subclase. La denominación de las fitocenosis se realizó también según dicho código. La caracterización de los rangos principales (clase, orden y alianza) a los que pertenecen las fitocenosis encontradas se presenta en Borhidi (1991, 1996). En la descripción de los estratos y sinucias se establecieron las siguientes categorías de presencia de las especies: constantes: presentes en el $80 \%$ o más de las listas; frecuentes: presentes del 60 al 79\%; menos frecuentes: presentes del 30 al 59\%; ocasionales: presentes del 15 al 29\%. Las especies con valores menores al $15 \%$ no fueron nombradas en la descripción. También se midió (cm) la estratificación de la hojarasca, se registró la existencia de raíces y raicillas y las características de la estera radical, si la hubiere.

Los nombres científicos completos (género, especie y autor) se encuentran en las tablas, como establece la Recomendación 7B del código anteriormente expuesto.

\section{Resultados}

De acuerdo al sistema jerárquico anteriormente expuesto en este trabajo se observan:

- Clase: Thrinaceto - Plumerietea obtusatae Borhidi 1996.

- Orden: Synapsidi ilicifoliae - Coccothrinacetalia Borhidi 1996.

- Alianza: Synapsidi ilicifoliae - Coccothrinacion Borhidi 1996.

Nuevas asociaciones estudiadas:

- Hohenbergio penduliflorae-Guapiretum obtusatae. - Rhipsali cassuthae-Hohenbergietum penduliflorae.

Hohenbergio penduliflorae - Guapiretum obtusatae Reyes \& Acosta ass. n.

Esta comunidad, constituida por un bosque siempreverde micrófilo con poca cobertura, es la predominante en la parte superior de los mogotes del Carso de Baire. Debido a su posición topográfica, esta fitocenosis se presenta sobre un microrelieve muy irregular, conocido como "lapiez", grande, agudo, con gran cantidad de huecos y diaclasas, donde ocasionalmente se encuentra un suelo denominado Rendzina roja, muy poco profundo. Generalmente en los huecos se acumula la hojarasca, a veces hasta $20 \mathrm{~cm}$, por lo que se conforma una estera radical. La altitud varía entre 500 y 670 msm.

Una característica peculiar de esta fitocenosis es que está integrada por especies xeromórficas y suculentas, además tiene fijadas al substrato y son dominantes muchas especies que en otros ecosistemas crecen como epífitas o lianas, entre ellas Hohenbergia penduliflora (A. Rich.) Mez, Tillandsia fasciculata Sw., Philodendron lacerum (Jacq.) Schott, Selenicerus grandiflorus (L.) Britt. \& Rose, etc., las que junto con Agave underwoodii Trelease y Oplonia tetrasticha (Wr. ex Griseb.) Stearn. conforman generalmente la mayor cobertura de los estratos inferiores, y que junto con los árboles de Guapira obtusata dan un aspecto particular.

El estrato arbóreo $\left(E_{3}\right)$ es irregular en altura, generalmente fluctúa entre 7 y $12 \mathrm{~m}$; su cobertura varía entre 50 y $70 \%$, excepcionalmente menos; las hojas son fundamentalmente xeromórficas y principalmente micrófilas. Como promedio entre las listas se hallan 11 especies, siendo constantes Guapira obtusata (abundante) y Plumeria emarginata Griseb.; como frecuente se observa a Tabebuia bibracteolata Griseb. Como menos frecuentes se encuentran Drypetes alba Poit., Amyris elemifera L., Coccothrinax elegans Borhidi \& Muñiz (a veces abundante), Alvaradoa arborescens Griseb., Hyperbaena paucinervis Urb. y Colubrina elliptica (Sw.) Brizicki \& Stern. Ocasionalmente se presentan Zanthoxylum cubense P. Wils., Drypetes mucronata Griseb., Spirotecoma sp., Savia bahamensis Britt., Exostema caribaea (Jacq.) R. \& S., Celtis trinervia Lam., Cameraria retusa Griseb., Gerascanthus gerascanthoides (K.) Borhidi y Cytharexylum caudatum L.

El estrato arbustivo $\left(\mathrm{E}_{2}\right)$ cubre generalmente entre 30 y $80 \%$ del área, a veces menos; tiene como promedio 11,6 especies. Como constante se observa a Ocotea coriacea (Sw.) Britt. y como frecuente a Guapira obtusata. Como menos frecuentes se hallan Plumeria emarginata, Hyperbaena paucinervis, Tabebuia bibracteolata, Amyris elemifera, Savia bahamensis, Alvaradoa arborescens y Phyllanthus epyphyllanthus L. La categoría más abundante es la de ocasionales, donde se encuentran Colubrina elliptica, Zanthoxylum cubense, Celtis trinervia, Capparis flexuosa L., Coccothrinax elegans, Erithalis fruticosa L., Thouinia trifoliata Poit., Caesalpinia vesicaria L., Erythroxylum rotundifolium Lunan, Drypetes alba Poit., D. mucronata y Exostema caribaea.

El estrato herbáceo $\left(\mathrm{E}_{1}\right)$ cubre entre 50 y $90 \%$, generalmente de 80 a $90 \%$ y tiene como promedio 25,4 especies. Las constantes son Agave underwoodii (abundante), Pilosocereus brooksianus 
(Vamp.) Bol. \& Row. y Selenicereus grandiflorus (a veces abundante); a su vez, las frecuentes son Hohenbergia penduliflora, Tillandsia fasciculata (abundantes), Coccothrinax elegans y Oplonia tetrasticha. Las demás, (muchas especies) son menos frecuentes, ocasionales o aún con menor presencia.

Las lianas son relativamente abundantes con 9,7 especies como promedio. Las constantes son Chiococca alba (L.) Hitchc. y Smilax havanensis Jacq., las que a veces son abundantes; a su vez, frecuentes son Platygyna dentata Alain y Stigmaphyllon sagreanum A. Juss. Como menos frecuentes se presentan Passiflora sexflora Juss., P. penduliflora Bert., Arthrostylidium capillifolium Griseb. (a veces abundante), Philodendron lacerum, Vanilla sp., Stigmaphyllon lineare Wr. y Cynanchum sp. Como ocasionales se hallan Solandra longiflora Tuss., Lasiasis divaricada (L.) Hitchc., Passiflora suberosa L. y Commicarpus scandens (L.) Standl.

En las epífitas, que son 6,7 como promedio, es constante y abundante Tillandsia fasciculata y a veces abundante $T$. usneoides L.; como frecuente se observa a $T$. recurvata $\mathrm{L}$. y como menos frecuentes $T$. pruinosa Sw., T. shiediana Stud., $T$. balbisiana Schult. y Epidendrum nocturnum Jacq. Como ocasional se encuentra a Guzmania monostachya (L.) Rusby ex Mez.
Esta fitocenosis, que fue descrita con nueve listas, tiene buena homogeneidad y sigue bien la ley de Raunkiaer, la combinación característica está compuesta por 27 especies (Tab. 1).

Se observan tres subasociaciones, que son: - Hohenbergio penduliflorae - Guapiretum obtusatae malpighietosum acunanae.

- Hohenbergio penduliflorae - Guapiretum obtusatae erythroxyletosum rotundifoliae.

- Hohenbergio penduliflorae - Guapiretum obtusatae phyllanthetosum epyphyllanthi.

Hohenbergio penduliflorae - Guapiretum obtusatae malpighietosum acunanae Reyes subass. n.

Esta fitocenosis se presenta en Las Manuelas, por lo que se halla a más baja altitud (500 msm) y con menor cantidad de lluvia. Faltan en ella Alvaradoa arborescens, Savia bahamensis y Epidendrum nocturnum que forman parte de la combinación característica. Es la subasociación más pobre en lianas y epífitas. Conforman la combinación diferencial varias especies. La lista tipo es la 26 (Tab. 1).

Hohenbergio penduliflorae - Guapiretum obtusatae erythroxyletosum rotundifoliae Reyes subass. n.

Ocupa las partes medias del área estudiada (La Tabla, Palma del Perro), con las condiciones ecológicas expuestas. Es la subasociación más rica en especies y

Tabla 1 - Hohenbergio penduliflorae-Guapiretum obtusatae en los mogotes del Carso de Baire. dp- lapiez o diente de perro, Presen- Presencia, L- lianas, Ep-.epífitas.

Table 1 - Hohenbergio penduliflorae-Guapiretum obtusatae in the mogotes of Carso de Baire. dp- lapiez or dogtooth, PresenConstancy, L- lianas, Ep- epyphytes.

\begin{tabular}{|c|c|c|c|c|c|c|c|c|}
\hline \multirow{2}{*}{$\begin{array}{l}\text { Subasociaciones } \\
\text { Lista Nr. }\end{array}$} & \multicolumn{2}{|c|}{$\begin{array}{l}\text { Malpighietosum } \\
\text { acunanae }\end{array}$} & \multicolumn{2}{|c|}{$\begin{array}{l}\text { Erythroxyletosum } \\
\text { rotundifoliae }\end{array}$} & \multicolumn{3}{|c|}{$\begin{array}{l}\text { Phyllanthetosum } \\
\text { epyphyllanthi }\end{array}$} & \multirow[t]{2}{*}{ Presen } \\
\hline & 27 & 26 & 2423 & $19 \quad 16$ & 5 & 4 & 3 & \\
\hline Altitud (msm) & 500 & 500 & 600620 & 670625 & 630 & 640 & 600 & \\
\hline Substrato & $\mathrm{dp}$ & $\mathrm{dp}$ & $d p \quad d p$ & $d p \quad d p$ & $\mathrm{dp}$ & $\mathrm{dp}$ & $\mathrm{dp}$ & \\
\hline $\begin{array}{l}\mathrm{E}_{33} \text { Estrato arbóreo } \\
(\% \text { cobertura })\end{array}$ & 20 & 60 & $60 \quad 60$ & $50 \quad 50$ & 50 & 60 & 60 & \\
\hline $\begin{array}{l}\mathrm{E}_{2-} \text { Estrato } \\
\text { arbustivo }(\%)\end{array}$ & 80 & 15 & $30 \quad 30$ & $60 \quad 10$ & 70 & 50 & 70 & \\
\hline $\begin{array}{l}\mathrm{E}_{1-} \text { Estrato } \\
\text { herbáceo }(\%)\end{array}$ & 70 & 80 & $90 \quad 80$ & $90 \quad 80$ & 80 & 80 & 50 & \\
\hline \multicolumn{9}{|c|}{ Combinación característica } \\
\hline $\begin{array}{l}\mathrm{E}_{3}-\text { Guapira obtusata } \\
\text { (Jacq.) Britt. }\end{array}$ & 2.1 & 2.1 & 2.13 .2 & 3.13 .2 & 3.1 & 3.1 & 3.1 & $\mathrm{~V}(2-3)$ \\
\hline $\begin{array}{l}\text { Coccothrinax elegans } \\
\text { Borhidi \& Muñiz }\end{array}$ & +.1 & 3.2 & $+.1 \mathrm{r} .1$ & +.12 .2 & +.1 & r. 1 & +.1 & $V(r-3)$ \\
\hline $\begin{array}{l}\text { Tabebuia bibracteolata } \\
\text { Griseb. }\end{array}$ & +.1 & 2.1 & $+.1+.1$ & $2.1 \mathrm{r} .1$ & 1.1 & r. 1 & 1.1 & $V(r-2)$ \\
\hline
\end{tabular}




\begin{tabular}{|c|c|c|c|c|c|c|c|c|c|}
\hline \multirow{3}{*}{$\begin{array}{l}\text { Subasociaciones } \\
\text { Plumeria emarginata Griseb. } \\
\text { Drypetes alba Poit. }\end{array}$} & \multicolumn{2}{|c|}{$\begin{array}{l}\text { Malpighietosum } \\
\text { acunanae }\end{array}$} & \multicolumn{3}{|c|}{$\begin{array}{l}\text { Erythroxyletosum } \\
\text { rotundifoliae }\end{array}$} & \multicolumn{3}{|c|}{$\begin{array}{l}\text { Phyllanthetosum } \\
\text { epyphyllanthi }\end{array}$} & \multirow{2}{*}{$\begin{array}{l}\text { Presen } \\
\mathrm{V}(\mathrm{r}-3)\end{array}$} \\
\hline & +.1 & 3.1 & $+.1+.1$ & 1.1 & 1.1 & . & r. 1 & r. 1 & \\
\hline & . & +.1 & $\mathrm{r} .1+.1$ & +.1 & +.1 & 2.1 & 2.1 & 1.1 & $\mathrm{~V}(\mathrm{r}-2)$ \\
\hline $\begin{array}{l}\mathrm{E}_{2}-\text { Ocotea coriacea } \\
\text { (Sw.) Britt. }\end{array}$ & +.1 & +.1 & $+.1 \mathrm{r} .1$ & r. 1 & r. 1 & 2.1 & +.1 & 2.1 & $\mathrm{~V}(\mathrm{r}-2)$ \\
\hline $\begin{array}{l}\mathrm{E}_{1}-\text { Agave underwoodii } \\
\text { Trelease }\end{array}$ & 3.2 & 1.2 & 4.23 .2 & 4.2 & 1.2 & 2.2 & 1.2 & . & $\mathrm{V}(1-4)$ \\
\hline $\begin{array}{l}\text { Oplonia tetrasticha } \\
\text { (Wr. ex Griseb.) Stearn. }\end{array}$ & 1.1 & 3.2 & 2.22 .2 & 3.2 & +.1 & 2.2 & 1.2 & 2.2 & $\mathrm{~V}(+-3)$ \\
\hline $\begin{array}{l}\text { Hohenbergia penduliflora } \\
\quad \text { (A. Rich.) Mez }\end{array}$ & 1.2 & 4.3 & +.23 .3 & 2.2 & 4.3 & +.2 & 3.2 & 2.2 & $\mathrm{~V}(+-4)$ \\
\hline Tillandsia fasciculata $\mathrm{Sw}$. & 2.2 & 2.2 & 2.23 .2 & 3.2 & 3.2 & 3.2 & 2.2 & 2.2 & $V(2-3)$ \\
\hline $\begin{array}{l}\text { Pilosocereus brooksianus } \\
\text { (Vamp.) Bol. \& Row. }\end{array}$ & 1.1 & 1.1 & $+.1 \mathrm{r} .1$ & +.1 & r. 1 & . & +.1 & r. 1 & $\mathrm{~V}(\mathrm{r}-1)$ \\
\hline $\begin{array}{l}\text { Philodendron lacerum } \\
\quad \text { (Jacq.) Schott }\end{array}$ & 1.1 & 1.1 & 1.11 .1 & 1.1 & 2.2 & r. 1 & r. 1 & +.1 & $\mathrm{~V}(\mathrm{r}-2)$ \\
\hline $\begin{array}{l}\text { Selenicerus grandiflorus } \\
\text { (L.) Britt. \& Rose }\end{array}$ & 1.1 & 1.1 & 1.12 .2 & 1.1 & 1.1 & +.1 & +.1 & +.1 & $\mathrm{~V}(+-2)$ \\
\hline L- Smilax havanensis Jacq. & 3.2 & 2.1 & $1.1+.1$ & r. 1 & +.1 & r. 1 & +.1 & r. 1 & $V(r-3)$ \\
\hline Chioccoca alba (L.) Hitchc. & 1.1 & +.1 & 1.11 .1 & 1.1 & +.1 & 1.1 & 1.1 & 2.1 & $\mathrm{~V}(+-2)$ \\
\hline Ep- Tillandsia usneoides L. & 1.2 & 1.2 & 1.23 .2 & 2.2 & +.2 & +.2 & . & +.2 & $\mathrm{~V}(+-3)$ \\
\hline $\begin{array}{l}\mathrm{E}_{3}-\text { Zanthoxylum cubense } \\
\text { P. Wils. }\end{array}$ & r. 1 & . & $+.1 \mathrm{r} .1$ & +.1 & r. 1 & . & r. 1 & r. 1 & $\operatorname{IV}(\mathrm{r}-+)$ \\
\hline Amyris elemifera $\mathrm{L}$. & . & r. 1 & 3.12 .1 & +.1 & . & 1.1 & 2.1 & r. 1 & $\operatorname{IV}(r-3)$ \\
\hline Hyperbaena paucinervis Urb. & +.1 & +.1 & $+.1 \mathrm{r} .1$ & . & 1.1 & r. 1 & . & 3.1 & $\operatorname{IV}(r-3)$ \\
\hline $\mathrm{E}_{1}-$ Vernonia sp. & +.1 & +.1 & 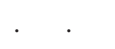 & r. 1 & r. 1 & . & r. 1 & r. 1 & $\mathrm{IV}(\mathrm{r}-+)$ \\
\hline $\begin{array}{l}\text { Grisebachianthus plucheoides } \\
\text { (Griseb.) King \& Robins. }\end{array}$ & +.1 & 2.2 & +.11 .1 & . & r. 1 & +.1 & . & r. 1 & $\mathrm{IV}(\mathrm{r}-2)$ \\
\hline L- Vanilla sp. & 1.1 & . & r. $1+.1$ & . & +.1 & r. 1 & r. 1 & . & $\operatorname{IV}(\mathrm{r}-1)$ \\
\hline $\begin{array}{l}\text { Stigmaphyllon sagreanum } \\
\text { A. Juss. }\end{array}$ & r. 1 & r. 1 & r. 1 r. 1 & r. 1 & +.1 & r. 1 & . & . & $\operatorname{IV}(\mathrm{r}-+)$ \\
\hline Platygyna dentata Alain & +.1 & 2.2 & r. 1 r. 1 & r. 1 & r. 1 & . & . & . & $\operatorname{IV}(r-2)$ \\
\hline Ep- Tillandsia recurvata $\mathrm{L}$. & +.1 & 1.2 & r. $1+.1$ & +.1 & r. 1 & . & . & . & $\operatorname{IV}(r-1)$ \\
\hline $\begin{array}{l}\mathrm{E}_{3}-\text { Alvaradoa arborescens } \\
\text { Griseb. }\end{array}$ & . & . & $+.1+.1$ & . & +.1 & r. 1 & +.1 & 2.1 & $\operatorname{IV}(r-2)$ \\
\hline $\mathrm{E}_{2}-$ Savia bahamensis Britt. & . & . & 2.21 .1 & 3.2 & +.1 & +.1 & +.1 & +.1 & $\operatorname{IV}(+-3)$ \\
\hline $\begin{array}{l}\mathrm{E}_{1}-\text { Epidendrum nocturnum } \\
\text { Jacq. }\end{array}$ & . & . & $1.2+.2$ & +.2 & +.2 & r. 2 & +.2 & +.2 & $\operatorname{IV}(r-1)$ \\
\hline \multicolumn{10}{|c|}{ Combinaciones diferenciales } \\
\hline $\begin{array}{l}\mathrm{E}_{3}-\text { Colubrina elliptica } \\
\text { (Sw.) Brizicki \& Stern. }\end{array}$ & 4.2 & 2.1 & $+.1 \mathrm{r} .1$ & +.1 & . & . & . & . & $\mathrm{III}(\mathrm{r}-4)$ \\
\hline $\mathrm{E}_{2}-$ Celtis trinervia Lam. & 1.1 & +.1 & r. 1 r. 1 & r. 1 & $\cdot$ & . & . & . & $\mathrm{III}(\mathrm{r}-1)$ \\
\hline $\mathrm{E}_{1}-$ Picramnia pentandra $\mathrm{Sw}$. & 1.1 & +.1 & r.1. & r. 1 & r. 1 & . & . & . & $\mathrm{III}(\mathrm{r}-1)$ \\
\hline $\begin{array}{l}\mathrm{E}_{3}-\text { Drypetes mucronata } \\
\text { Griseb. }\end{array}$ & . & . & $+.1+.1$ & +.1 & 2.1 & . & . & . & $\mathrm{III}(+-2)$ \\
\hline $\begin{array}{l}\mathrm{E}_{2}-\text { Erythroxylum } \\
\text { rotundifolium Lunan }\end{array}$ & . & . & $+.1 \mathrm{r} .1$ & r. 1 & +.1 & . & . & r. 1 & $\mathrm{III}(\mathrm{r}-+)$ \\
\hline Erithalis fruticosa L. & . & . & $\mathrm{r} .1+.1$ & r. 1 & . & . & . & 1.1 & $\operatorname{III}(\mathrm{r}-+)$ \\
\hline $\begin{array}{l}\mathrm{E}_{1}-\text { Polypodium } \\
\text { polypodioides (L.)A.S. Hith. }\end{array}$ & . & . & r. $2+.2$ & r. 2 & +.2 & . & . & . & $\operatorname{III}(\mathrm{r}-+)$ \\
\hline $\begin{array}{l}\text { Epidendrum pygmaeum } \\
\text { Hook. }\end{array}$ & . & . & +.21 .2 & 2.2 & 1.2 & . & . & r. 1 & $\mathrm{III}(+-2)$ \\
\hline $\begin{array}{l}\text { L- Passiflora penduliflora } \\
\text { Bert. }\end{array}$ & . & . & $\mathrm{r} .1+.1$ & r. 1 & r. 1 & . & . & . & $\mathrm{III}(\mathrm{r}-+)$ \\
\hline
\end{tabular}




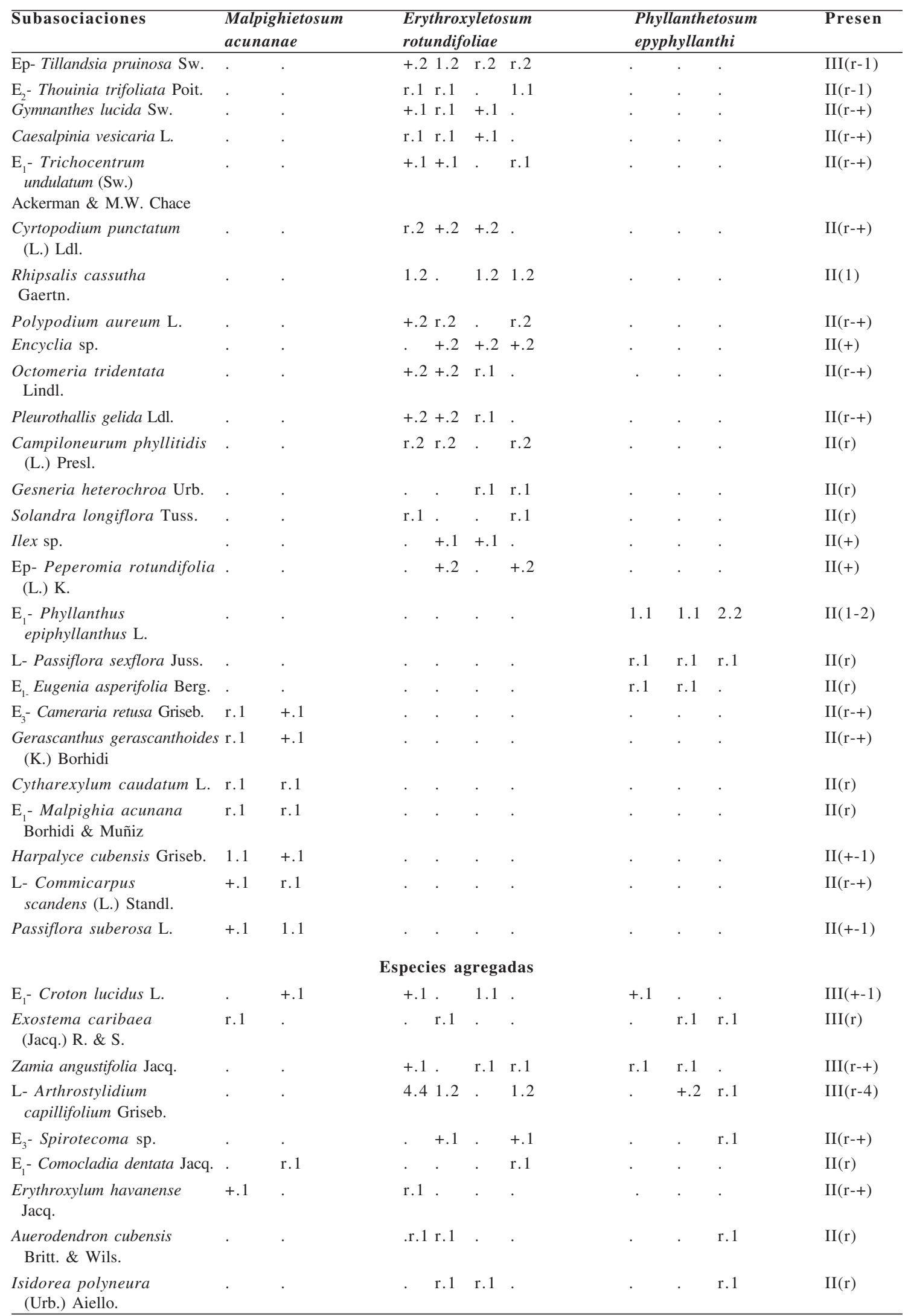




\begin{tabular}{|c|c|c|c|c|c|c|c|c|c|c|}
\hline Subasociaciones & $\begin{array}{l}M a l \\
a c u\end{array}$ & $\begin{array}{l}\text { hietosum } \\
\text { lae }\end{array}$ & $\begin{array}{l}\text { Ery } \\
\text { rott }\end{array}$ & throx & $\begin{array}{l}\text { leto } \\
\text { liae }\end{array}$ & sum & & $\overrightarrow{a l}$ & $\begin{array}{l}\text { tosum } \\
\text { thi }\end{array}$ & Presen \\
\hline Pharus glaber $\mathrm{K}$. & r. 1 & . & r. 1 & . & . & +.1 & . & . & . & $\mathrm{II}(\mathrm{r}-+)$ \\
\hline $\begin{array}{l}\text { Peperomia magnoliaefolia } \\
\text { (Jacq.) A. Dietr. }\end{array}$ & . & . & r. 1 & . & . & . & r. 1 & . & . & $\mathrm{II}(\mathrm{r})$ \\
\hline L- Capparis flexuosa $\mathrm{L}$. & . & +.1 & . & r. 1 & . & +.1 & . & . & . & $\mathrm{II}(\mathrm{r}-+)$ \\
\hline Cynanchum sp. & . & r. 1 & r. 1 & . & +.1 & . & . & . & . & $\mathrm{II}(\mathrm{r}-+)$ \\
\hline Stigmaphyllon lineare $\mathrm{Wr}$. & . & r. 1 & . & . & . & +.1 & r. 1 & . & . & $\mathrm{II}(\mathrm{r}-+)$ \\
\hline $\begin{array}{l}\text { Lasiasis divaricata } \\
\text { (L.) Hitchc. }\end{array}$ & +.1 & . & . & r.1. & . & . & . & . & . & $\mathrm{II}(\mathrm{r}-+)$ \\
\hline $\begin{array}{l}\text { Ep- Guzmania monostachya } \\
\text { (L.) Rusby ex Mez }\end{array}$ & & . & r. 1 & . & . & r. 1 & r. 1 & . & . & $\mathrm{II}(\mathrm{r})$ \\
\hline Tillandsia schiediana Stud. & . & r. 1 & . & . & +.1 & r. 1 & . & . & . & $\mathrm{II}(\mathrm{r}-+)$ \\
\hline T. balbisiana Schult. & . & +.1 & r. 1 & +.1 & . & . & . & . & . & $\mathrm{II}(\mathrm{r}-+)$ \\
\hline
\end{tabular}

Especies en una sola lista. Lista 3.Jacquinia roigii P. Wils. r.1, Erythroxylum sp.r.1, Garcinia aristalata (Griseb.) Borhidi +.1, Pleurothalis sertularioides (Sw.) Spreng. + .2; Lista 4. Tillandsia bulbosa Hook. r.1; Lista 5. Salvia sp. r.1,Clusia rosea Jacq.r.1,Adiantum trapeziforme L.r.1, Garrya fadyenii Hook. r.1,Aechmea mudicaulis (L.) Griseb. +.1; Lista 16.Zizyphus rhodoxylon Urb. 1.1, Ficus laevigata Vahl. r.1,Urera baccifera (L.) Gaud. r.1, Coelia triptera (Sw.) D. Donr.1, Maxillaria sp.r.1, Encyclia sp. 1 r.1, Pilea sp. 1+.2,Rhacoma sp. (r.1), Psychotria sp.r.1,Peperomia sp. 1 r.1,Anthurium cubense Engler r.2,Philodendron krebsii Schottr.1; Lista 19. Solanum sp.r.1,Dendropanax arboreus Dene. \& Planch. r.1, Peperomia sp.r.1;Lista23.Ficus perforata L.r.1,Rhacoma sp.+.1,Peperomia sp. 2+.1, Crotonsp. 1.2,Catopsis sp.r.1,Encycliasp. 2+.1;Lista 24. Oeceoclades maculata (Ldl.) Ldl. r.2, Guettarda sp.r.1,Pilea sp. 2 r.1; Lista 26.Pentalinum luteum (L.) Hanse \& Wunderlin +.1, Passiflora sp.r.1; Lista 27. Faramea occidentalis (L.) A. Rich. r.1, Guettarda sp. r.1

Species in a single list. List 3. Jacquinia roigii P. Wils. r.1, Erythroxylum sp. r.1, Garcinia aristalata (Griseb.) Borhidi +.1, Pleurothalis sertularioides (Sw.) Spreng. +.2; List 4. Tillandsia bulbosa Hook. r.1; Lista 5. Salvia sp. r.1, Clusia rosea Jacq. r.1, Adiantum trapeziforme L. r.1, Garrya fadyenii Hook. r.1, Aechmea nudicaulis (L.) Griseb. +.1; List 16.Zizyphus rhodoxylon Urb. 1.1, Ficus laevigata Vahl. r.1, Urera baccifera (L.) Gaud. r.1, Coelia triptera (Sw.) D. Don r.1, Maxillaria sp. r.1, Encyclia sp. 1 r.1, Pilea sp. 1 +.2, Rhacoma sp. (r.1), Psychotria sp. r.1, Peperomia sp. 1 r.1, Anthurium cubense Engler r.2, Philodendron krebsii Schott r.1; List 19. Solanum sp. r.1, Dendropanax arboreus Dene. \& Planch. r.1, Peperomia sp. r.1;

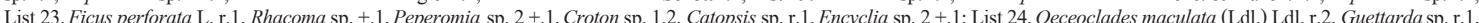
Pilea sp. 2 r.1; List 26. Pentalinum luteum (L.) Hanse \& Wunderlin +.1, Passiflora sp. r.1; List 27. Faramea occidentalis (L.) A. Rich. r.1, Guettarda sp. r.1.

la que tiene una combinación diferencial más prolífica. Se presenta aquí la mayor riqueza en el estrato herbáceo, lianas y epífitas. La lista tipo para esta subasociación y para la asociación es la 23 (Tab. 1).

- Hohenbergio penduliflorae - Guapiretum obtusatae phyllanthetosum epyphyllanthi Reyes subass. n.

Esta subasociación se halla en la parte Sur del territorio estudiado, próxima a las más importantes altitudes, por ello recibe mayor cantidad de lluvia. Es la más pobre en especies y faltan incluso Platygyna dentata y Tillandsia recurvata que forman parte de la combinación característica; tiene a su vez la menor cuantía de especies en los estratos arbustivo $(8,6)$, herbáceo (14) y lianas (7). Se destaca la abundancia de Drypetes alba y Ocotea coriacea. La combinación diferencial es pequeña. La lista tipo es la cuatro (Tab. 1).

Rhipsali cassuthae - Hohenbergietum penduliflorae Reyes \& Acosta ass. n.

Esta asociación se presenta en las paredes de los mogotes, las que tienen una inclinación de alrededor de 90 grados. Debido a dicha inclinación, no se encuentra suelo, sino las plantas crecen fijadas a las fisuras de las rocas, por lo que es una comunidad muy característica, tanto fisonómica como ecológicamente, pues crece en condiciones muy extremas, sobre todo edáficas. La variación de altitud entre las listas es mínima, entre 570 y 600 msm.

Esta fitocenosis, que fue descrita con seis listas, conforma un matorral entre 0,80 y $1,20 \mathrm{~m}$ de altura. El subestrato de arbustos mayores tiene una cobertura entre 5 y $20 \%$, excepcionalmente más, a veces alcanzan de 2 a $3 \mathrm{~m}$; su promedio de especies por lista es de 8,7 .

El subestrato inferior, que cubre de 60 a $80 \%$, tiene dicho promedio de 24,1 . Su fisonomía es muy peculiar, pues predominan especies de tipo rosulado y suculentas; muchas de ellas están aquí fijadas al substrato rocoso, mientras que en otros ecosistemas se presentan solo como epífitas, entre ellas Hohenbergia penduliflora, Tillandsia fasciculata, Rhipsalis cassutha Gaertn., Trichocentrum undulatum (Sw.) Ackerman \& M. W. Chace, Marcgravia rectiflora Tr. \& Pl., etc.

Esta fitocenosis es homogénea y sigue bien la ley de Raunkiaer, la combinación característica está compuesta por 27 especies (Tab. 2).

Se observan dos subasociaciones que son: - Rhipsali cassuthae - Hohenbergietum penduliflorae pilocereetosum brooksiani.

- Rhipsali cassuthae - Hohenbergietum penduliflorae typicum.

Rhipsali cassuthae - Hohenbergietum penduliflorae pilocereetosum brooksiani Reyes subass. n.

Esta subasociación se presenta en el interior del conjunto de mogotes, frente a los valles interiores. 
Por ello, el movimiento del viento es menor; además, debido a la estrechez de los valles y la contigüidad de los mogotes, la insolación es más pequeña. Se halla una combinación diferencial bien desarrollada (Tab. 2). La lista tipo es la 13.

Rhipsali cassuthae - Hohenbergietum penduliflorae typicum Reyes subass. $\mathrm{n}$.
Se encuentra en la parte exterior de los mogotes, donde hay más movimiento del viento, mayor insolación durante las tardes y por tanto calentamiento de las calizas. Ello produce un aumento de la evapotranspiración y con ello un estrés hídrico más acentuado. La lista tipo para esta subasociación y para la asociación es la 21. Se hallan

Tabla 2 - Rhipsali cassuthae-Hohenbergietum penduliflorae en los paredones de los mogotes del Carso de Baire. Table 2 - Rhipsali cassuthae-Hohenbergietum penduliflorae in the wall of the mogotes of Carso de Baire.

\begin{tabular}{|c|c|c|c|c|c|c|c|}
\hline \multirow{2}{*}{$\begin{array}{l}\text { Subasociación } \\
\text { Variantes }\end{array}$} & \multicolumn{3}{|c|}{ Typicum } & \multicolumn{3}{|c|}{ Pilosocereetosum brooksiani } & \multirow[t]{2}{*}{ Presen } \\
\hline & Típica & \multicolumn{5}{|c|}{$\begin{array}{l}\text { Plumeria } \\
\text { emarginata }\end{array}$} & \\
\hline Lista Nr. & 9 & 21 & 10 & 13 & 14 & 17 & \\
\hline Altitud (msm) & 580 & 570 & 600 & 575 & 570 & 600 & \\
\hline Inclinación (grados) & 90 & 90 & 90 & 90 & 90 & 90 & \\
\hline Exposición & $\mathrm{W}$ & W & $\mathrm{N}$ & ENE & W & W & \\
\hline $\begin{array}{l}\mathrm{E}_{2-} \text { Estrato arbustivo } \\
(\% \text { cobertura })\end{array}$ & 5 & 50 & 5 & 20 & 10 & 10 & \\
\hline $\mathrm{E}_{1-}$ Estrato herbáceo (\%) & 80 & 80 & 60 & 70 & 70 & 60 & \\
\hline \multicolumn{8}{|c|}{ Combinación característica } \\
\hline Agave underwoodii Trelease & 4.2 & 2.2 & 1.2 & 2.2 & 1.2 & 2.2 & $V(1-4)$ \\
\hline Rhipsalis cassutha Gaertn. & 2.2 & 3.2 & . & 1.2 & 2.2 & 3.2 & $\mathrm{~V}(1-3)$ \\
\hline $\begin{array}{l}\text { Hohenbergia penduliflora } \\
\text { (A. Rich.) Mez }\end{array}$ & . & 3.2 & +.2 & 1.2 & 3.3 & 2.2 & $V(+-3)$ \\
\hline Tillandsia fasciculata Sw. & 2.2 & 2.2 & 1.2 & 1.2 & 2.2 & 1.2 & $\mathrm{~V}(1-2)$ \\
\hline $\begin{array}{l}\text { Marcgravia rectiflora } \\
\text { Tr. \& Pl. }\end{array}$ & 2.2 & +.2 & . & 3.3 & 2.2 & +.2 & $\mathrm{~V}(+-3)$ \\
\hline $\begin{array}{l}\text { Pitcairnia cubensis } \\
\text { (Mez) L. B. Smith }\end{array}$ & +.2 & +.2 & 2.2 & 1.2 & 1.2 & 2.2 & $V(+-2)$ \\
\hline $\begin{array}{l}\text { Grisebachianthus plucheoides } \\
\text { (Griseb.) King \& Robins. }\end{array}$ & r. 1 & r.1 & +.1 & 1.1 & +.1 & +.1 & $\mathrm{~V}(\mathrm{r}-1)$ \\
\hline Ficus laevigata Vahl. & r. 1 & 3.1 & r.1 & +.1 & +.1 & +.1 & $\mathrm{~V}(\mathrm{r}-3)$ \\
\hline Picramnia pentandra Sw. & r. 1 & 1.1 & r. 1 & +.1 & 1.1 & +.1 & $\mathrm{~V}(\mathrm{r}-1)$ \\
\hline Eupatorium $s p$ & 1.1 & 1.1 & +.1 & 1.1 & +.1 & . & $\mathrm{V}(+-1)$ \\
\hline $\begin{array}{l}\text { Rhytidophyllum mogoticola } \\
\text { Borhidi \& Muñiz }\end{array}$ & r.1 & 1.1 & . & +.1 & 1.1 & 2.1 & $\mathrm{~V}(\mathrm{r}-2)$ \\
\hline Guattarda calyptrata A. Rich. & r. 1 & . & r. 1 & r. 1 & +.1 & r.1 & $\mathrm{V}(\mathrm{r}-+)$ \\
\hline $\begin{array}{l}\text { Trichocentrum undulatum (Sw.) } \\
\text { Ackerman \& M. W. Chace }\end{array}$ & 1.2 & r.1 & r.1 & r. 2 & +.1 & +.2 & $\mathrm{~V}(\mathrm{r}-1)$ \\
\hline Adiantum tenerum $\mathrm{Sw}$. & r.2 & +.2 & . & +.2 & +.2 & 1.2 & $\mathrm{~V}(\mathrm{r}-1)$ \\
\hline $\begin{array}{l}\text { Polypodium polypodioides } \\
\text { (L.) A. S. Hitchc. }\end{array}$ & +.2 & +.2 & $\mathrm{r} .2$ & +.2 & . & +.2 & $\mathrm{~V}(\mathrm{r}-+)$ \\
\hline $\begin{array}{l}\text { Campiloneurum phyllitidis } \\
\text { (L.) Presl. }\end{array}$ & $\mathrm{r} .2$ & +.2 & . & r. 2 & r. 2 & 2.2 & $\mathrm{~V}(\mathrm{r}-2)$ \\
\hline $\begin{array}{l}\text { Philodendron lacerum } \\
\text { (Jacq.) Schott }\end{array}$ & 1.1 & 2.2 & +.1 & 1.2 & 2.1 & +.1 & $\mathrm{~V}(+-2)$ \\
\hline
\end{tabular}




\begin{tabular}{|c|c|c|c|c|c|c|c|}
\hline \multirow{2}{*}{$\begin{array}{l}\text { Subasociación } \\
\text { Chiococa alba }(\text { L.) Hitchc. }\end{array}$} & \multicolumn{3}{|c|}{ Typicum } & \multicolumn{3}{|c|}{ Pilosocereetosum brooksiani } & \multirow{2}{*}{$\frac{\text { Presen }}{\mathrm{V}(\mathrm{r}-+)}$} \\
\hline & . & +.1 & r.1 & r.1 & +.1 & +.1 & \\
\hline Tillandsia usneoides L. & +.2 & +.2 & r. 2 & $\mathrm{r} .2$ & r. 1 & 1.2 & $\mathrm{~V}(\mathrm{r}-1)$ \\
\hline $\begin{array}{l}\text { Guzmania monostachya }(\mathrm{L} .) \\
\text { Rusby ex Mez }\end{array}$ & r. 1 & +.1 & . & +.2 & r. 1 & $\mathrm{r} .2$ & $\mathrm{~V}(\mathrm{r}-+)$ \\
\hline $\begin{array}{l}\text { Coccothrinax elegans Borhidi } \\
\text { \& Muñiz }\end{array}$ & $\cdot$ & . & 2.2 & r. 1 & r. 1 & +.1 & $\operatorname{IV}(r-2)$ \\
\hline Phyllanthus epiphyllanthus L. & . & +.1 & 2.1 & 2.2 & +.1 & . & $\operatorname{IV}(+-2)$ \\
\hline $\begin{array}{l}\text { Oplonia tetrasticha } \\
\text { (Wr. ex Griseb.) Stearn. }\end{array}$ & . & r. 1 & r. 1 & . & +.1 & r. 1 & $\operatorname{IV}(\mathrm{r}-1)$ \\
\hline Gesneria heterochroa $U r b$. & . & 1.1 & 1.1 & +.1 & . & +.1 & $\operatorname{IV}(+-1)$ \\
\hline Hyperbaena paucinervis Urb. & . & 1.1 & $\mathrm{r} .1$ & r. 1 & r. 1 & . & $\operatorname{IV}(\mathrm{r}-1)$ \\
\hline Begonia sp. & . & 1.2 & r. 1 & r. 1 & . & 1.2 & $\operatorname{IV}(\mathrm{r}-1)$ \\
\hline $\begin{array}{l}\text { Selenicereus grandiflorus (L.) } \\
\text { Britt. \& Rose }\end{array}$ & $\cdot$ & +.1 & r. 1 & . & 2.2 & 1.1 & $\operatorname{IV}(\mathrm{r}-2)$ \\
\hline Ocotea coriacea (Sw.) Britt. & . & 2.1 & . & 1.1 & 1.1 & +.1 & $\operatorname{IV}(+-2)$ \\
\hline \multicolumn{8}{|c|}{ Combinación diferencial } \\
\hline $\begin{array}{l}\text { Pilosocereus brooksianus } \\
\text { (Vamp.) Byl. \& Row. }\end{array}$ & . & . & . & r.1 & 1.1 & r. 1 & $\mathrm{III}(\mathrm{r}-1)$ \\
\hline Hamelia patens Jacq. & . & . & . & r. 1 & r. 1 & r. 1 & III(r) \\
\hline $\begin{array}{l}\text { Cupania glabra Sw. var. } \\
\text { glabra }\end{array}$ & . & . & . & r. 1 & r. 1 & +.1 & $\mathrm{III}(\mathrm{r}-+)$ \\
\hline $\begin{array}{l}\text { Trichostigma octandrum (L.) } \\
\text { H. Walt. }\end{array}$ & . & . & . & r. 1 & 2.2 & r. 1 & $\mathrm{III}(\mathrm{r}-2)$ \\
\hline Coccoloba $s p$ & . & . & . & r. 1 & . & r. 1 & $\mathrm{II}(\mathrm{r})$ \\
\hline Pisonia aculeata $\mathrm{L}$. & . & . & . & r.1 & . & r.1 & $\mathrm{II}(\mathrm{r})$ \\
\hline Pilea $s p$ & . & . & . & $\mathrm{r} .2$ & +.2 & . & $\mathrm{II}(\mathrm{r}-+)$ \\
\hline Plumeria emarginata Griseb. & . & +.1 & r. 1 & . & . & . & $\mathrm{II}(\mathrm{r}-+)$ \\
\hline Epidendrum pygmaeum Hook. & . & +.2 & +.2 & . & . & . & $\mathrm{II}(+)$ \\
\hline \multicolumn{8}{|c|}{ Especies agregadas } \\
\hline Guapira obtusata (Jacq.) Britt. & & +.1 & +.1 & +.1 & . & . & $\mathrm{III}(+)$ \\
\hline Anthurium cubense Engler & . & $\mathrm{r} .1$ & . & r. 2 & r.1 & . & $\mathrm{III}(\mathrm{r})$ \\
\hline $\begin{array}{l}\text { Stigmaphyllon sagreanum } \\
\text { A. Juss. }\end{array}$ & +.1 & r. 1 & . & +.1 & . & . & $\mathrm{III}(\mathrm{r}-+)$ \\
\hline $\begin{array}{l}\text { Cissus verticillata }(\mathrm{L} .) \text { D. H. } \\
\text { Nicolson \& C. Jarvis }\end{array}$ & . & 2.2 & . & r. 1 & +.1 & . & $\mathrm{III}(\mathrm{r}-2)$ \\
\hline Tillandsia schiediana Stud. & r.1 & . & r. 2 & . & . & +.1 & $\mathrm{III}(\mathrm{r}-+)$ \\
\hline $\begin{array}{l}\text { Stigmaphyllon ledifolium } \\
\text { (K.) Small }\end{array}$ & . & r. 1 & . & r. 1 & . & . & $\mathrm{II}(\mathrm{r})$ \\
\hline Smilax havanensis Jacq. & . & . & +.1 & +.1 & . & . & $\mathrm{II}(+)$ \\
\hline Lepanthes sp. & . & . & r. 2 & . & +.2 & . & $\mathrm{II}(\mathrm{r}-+)$ \\
\hline Tillandsia pruinosa $\mathrm{Sw}$. & . & . & r.2 & $\mathrm{r} .2$ & . & . & $\mathrm{II}(\mathrm{r})$ \\
\hline Ficus perforata $\mathrm{L}$. & . & . & r. 1 & +.1 & . & . & $\mathrm{II}(\mathrm{r}-+)$ \\
\hline $\begin{array}{l}\text { Dendropanax arboreus (L.) } \\
\text { Dene. \& Planch. }\end{array}$ & . & r. 1 & . & r. 1 & . & . & $\mathrm{II}(\mathrm{r})$ \\
\hline Urera baccifera (L.) Gaud. & . & +.1 & . & . & r.1 & . & $\mathrm{II}(\mathrm{r}-+)$ \\
\hline
\end{tabular}




\begin{tabular}{|c|c|c|c|c|c|c|}
\hline Subasociación & & Typicum & & Pilos & tosum brooksiani & Presen \\
\hline Epidendrum nocturnum Jacq. & . & r.1 & . & r.1 & . & $\mathrm{II}(\mathrm{r})$ \\
\hline $\begin{array}{l}\text { Nephrolepis multiflora (Robx.) } \\
\text { Jarvet ex Morton }\end{array}$ & & +.2 & - & . & r. 2 & $\mathrm{II}(\mathrm{r}-+)$ \\
\hline Zamia angustifolia Jacq. & . & r. 2 & . & . & r. 2 & II(r) \\
\hline Vanilla sp. & +.1 & . & . & +.1 & . & II $(+)$ \\
\hline
\end{tabular}

Lista 9. Solandra longiflora Juss. r.1, Clusia rosea Jacq. 1.1, Catopsis sp. r.1; Lista 21. Tabebuia bibracteolata (Griseb.) Britt. +.1; Lista 10. Ficus citrifolia P. Mill. r.1, Vernonia sp.r.1, Amyris elemifera L. r.1, Platygyna dentata Alain r.1, Peperomia sp. r.1; Lista 13. Pharus glaber K. +.2, Faramea occidentalis (L.) A. Rich. r.1, Arthrostylidium capillifolium Griseb. 1.2,Smilax lanceolata L. r.1, Pilea sp. 1 r.1; Lista. 14. Cedrela odorata L.r.1, Passiflora suberosa L.r.1, Philodendron krebsii Schott r.1, Serjania sp. r.1, Tournefotia glabra L. r.1, Trophis racemosa (L.) Urb.r.1, Casearia hyrsuta Sw.r.1, Polypodium aureum L.r.2, Aristolochia sp r.1; Lista 17. Adiantum trapeziforme L. 1.2, Pilea sp. $2+.1$, Rhacoma sp.r.1, Vitis tiliaefolia H. \& B. r.1., Passiflora sp. r.1, Maxillaria sp. r.2, Oxandra lanceolata (Sw.) Baill. r.1.

List 9. Solandra longiflora Juss. r.1, Clusia rosea Jacq. 1.1, Catopsis sp. r.1; List 21. Tabebuia bibracteolata (Griseb.) Britt. +.1; List 10. Ficus citrifolia P. Mill. r.1, Vernonia sp. r.1, Amyris elemifera L. r.1, Platygyna dentata Alain r.1, Peperomia sp. r.1; List 13. Pharus glaber K. +.2, Faramea occidentalis (L.) A. Rich. r.1, Arthrostylidium capillifolium Griseb.

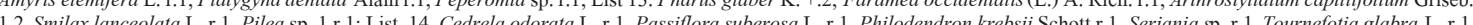

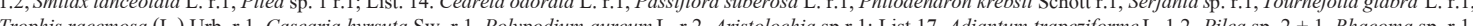

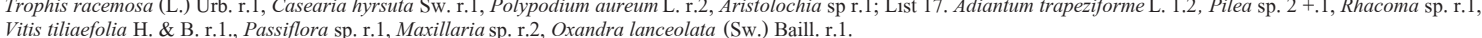

dos variantes, Plumeria emarginata y Típica; la primera, compuesta por las listas 10 y 21 , tiene como combinación diferencial a Plumeria emarginata y Epidendrum pygmaeum Hook.; en la segunda (lista 9) faltan diversas especies que forman parte de la combinación característica (Tab. 2).

\section{Discusión}

Las asociaciones encontradas concuerdan con la alianza Synapsidi ilicifoliae Coccothrinacion Borhidi descrita por Borhidi (1996), el que separó en esta zona la asociación Coccothrinaci - Tabebuietum bibracteolatae Borhidi \& Muñiz 1991.

En otras zonas cársicas montanas de Cuba Oriental y Cuba Central hay comunidades parecidas fisonómicamente, pero florísticamente diferentes, aunque corresponden a la alianza expuesta; en las de Cuba Occidental la disimilitud llega al nivel de órdenes (Borhidi 1991, 1996). En otras áreas con dicha geomorfología, pero de baja altitud de Cuba Oriental se observan otras fitocenosis, que pertenecen a otras alianzas u órdenes (Borhidi 1996; Reyes \& Acosta 2004 a; Reyes \& Martínez 2005), ya que hay otras condiciones ecológicas, o sea, mayores temperaturas y duración del período poco lluvioso. En los mogotes del norte de la República Dominicana, aunque respecto a la vegetación son parecidos fisonómicamente, desde el punto de vista florístico divergen notablemente (De los Ángeles et al. 2005; Zanoni et al. 1990) y son relativamente comparables a las áreas intramogotes en esta zona (Reyes \& Acosta 2004 b).

Como estas mesetas están fuertemente carsificadas (Viña 1991) y con gran percolación del principal substrato (calizas), la parte superior está compuesta por un lapiez muy agudo, donde generalmente no se presenta suelo, sino las oquedades se llenan de material vegetal en diversos estados de descomposición. Como la lluvia es frecuente (Gagua et al. 1989) y la humedad relativa es alta, se conforma una estera radical, embebida en una matriz de humus, subsistema tropical ya conocido (Herrera \& Rodríguez 1988; Reyes \& Acosta 2005; Matos 2007; Fornaris 2007) y donde se produce el reciclaje de nutrientes de la asociación Hohenbergio penduliflorae - Guapiretum obtusatae. Las diferencias entre las subasociaciones de dicha fitocenosis se deben fundamentalmente a las disimilitudes pluviométricas y geográficas, ya que ocupan áreas separadas con desigualdades altitudinales y en la lluvia recibida.

Rhipsali cassuthae - Hohenbergietum penduliflorae, como se desarrolla en paredes prácticamente verticales, utilizando las grietas existentes, las condiciones del substrato son extremas, características también observadas en algunas áreas entre terrazas costeras (Reyes 2004). No obstante, el subestrato inferior es relativamente rico en especies; las diferencias entre sus subasociaciones dependen fundamentalmente de la economía hídrica, ya que ocupan distintas posiciones respecto a la insolación y al viento.

Como inferencia de la inspección a las condiciones del substrato de las muestras y otros sitios de la zona de estudio, se considera que debido a la particularidad geológica y al lugar de desarrollo en los mogotes, estos ecosistemas están sometidos a un gran estrés hídrico y nutrimental, con combinaciones florísticas propias, por lo que se constituyen en únicos y deben ser utilizados como objetos de conservación prioritarios en la fundamentación de la protección del territorio. 


\section{Agradecimientos}

Al proyecto nacional Diversidad biológica de los macizos montañosos Sierra Maestra y Nipe Sagua Baracoa, en cuyo marco se realizó este estudio. A Eddy Martínez Quesada y María del Carmen Fagilde Espinosa por la determinación de las especies desconocidas, a Ángel Motito Marín por la revisión del manuscrito y a Pedro Bergues Garrido por la traducción al inglés del resumen.

\section{Referencias}

Barkman, J.J.; Moravec, J. \& Rauschert, S. 1988. Código de Nomenclatura Fitosociológica. 2 Ed. Opusc. Bot. Pharm. Complutensis 4: 9-61.

Borhidi, A. 1991. Phytogeography and vegetation ecology of Cuba. Akadémiai Kiadó, Budapest. 858p.

Borhidi, A. 1996. Phytogeography and vegetation ecology of Cuba. 2 Ed. Akadémiai Kiadó, Budapest. 926p.

Braun Blanquet, J. 1921. Prinzipien einer Systematik der Pfanzengeselschaften auf floristischer Grundlage. Jahrb. St. Gellener Naturw. Ges. 37.

Braun Blanquet, J. 1951. Pflanzensoziologie; Grundzüge der Vegetationskunde. 2 Aufl. Wien. 531p.

Braun Blanquet, J. 1964. Pflanzensoziologie; Grundzüge der Vegetationskunde. 3 Aufl. Wien, N. York. 637p.

De los Ángeles, I.; Clase, T. \& Peguero, B. 2005. Flora y vegetación del Parque Nacional El Choco, Sosua, provincia Puerto Plata, República Dominicana. Moscosoa 14: 10-55.

Fornaris, E. 2007. Estudio funcional de algunas formaciones boscosas de la Sierra Maestra. Tesis en Opción al Título Académico de Master en Ecología y Sistemática Aplicada, Mención Ecología. IES-BIOECO. 80p.

Gagua, G.; Zarembo, S. \& Izquierdo, A. 1989. Precipitación media anual. 1931-72. In: Nuevo Atlas Nacional de Cuba. Mapa 42. Pp. VI.3.1.

Greig-Smith, P. 1964. Quantitative plant ecology. 2 Ed. Butter Worths, London. 256p.

Herrera, R.A. \& Rodríguez, M. 1988. Clasificación funcional de los bosques tropicales. In: Ecología de los bosques siempreverdes de la Sierra del Rosario, Cuba. Montevideo, ROSTLAC. Pp. 574-626.

Iturralde Vinent, M.A. 2007. Geología de Cuba para todos. Cap. 5, Tectónica de Placas. Iturralde-Vinent, M.A. (ed.). Museo Nacional de Historia Natural-CITMA. Pp. 39-44.

Lapinel, B. 1989. Temperatura media anual del aire. En: Nuevo Atlas Nacional de Cuba. Mapa 15. Pp. VI.2.4.

López, A. 1998. Algunas características del endemismo de la flora de Cuba Oriental. In: Halfter, G. (comp.). La diversidad biológica de Iberoamérica II. Volumen Especial. Acta Zoológica Mexicana. Inst. Ecología, AC. Xalapa, México. Pp. 47-82.
López, A. 2005. Nueva perspectiva para la regionalización fitogeográfica de Cuba: definición de los sectores. In: Regionalización biogeográfica en Iberoamérica y tópicos afines. UNAM: 417-428.

Matos, R. 2007. Funcionamiento ecológico de la pluvisilva submontana sobre suelos de mal drenaje de Monte Iberia. Tesis en opción al título de Ingeniero Forestal. Ministerio de Educación Superior. Centro Universitario de Guantánamo. Facultad Agroforestal de Montaña. 41p.

Montenegro, U. 1991. Humedad relativa media anual. In: Atlas de Santiago de Cuba. Academia de Ciencias de Cuba. Mapa 28

Núñez, A. \& Viña, N. 1989. Regiones naturales antrópicas. In: Nuevo Atlas Nacional de Cuba. Pp. XII.2.1.

Núñez, A.; Viña, N. \& Graña, A. 1989. Carsología. In: Nuevo Atlas Nacional de Cuba. Mapa 1. Pp. V.1.2-3.

Oro, J.R. 1989. Evolución paleo geológica. In: Nuevo Atlas Nacional de Cuba. Pp. III.1.4.

Raunkiaer, C. 1934. The life forms of plants and statistical plant geography. Oxford University Press, Oxford. 632p.

Reyes, O.J. 2000. Las cuencas de los ríos Toa y Duaba como parte de la región Moa-Baracoa; su importancia en el desarrollo de la flora cubana. Ed. Academia. Biodiversidad de Cuba Oriental 5: 50-57.

Reyes, O.J. 2004. Fitocenosis presentes en las áreas costeras del sur de la Sierra Maestra. III. Asociación Tabebuio myrtifoliae - Colubrinetum ellipticae. Foresta Veracruzana 6: 7-14.

Reyes, O.J. \& Acosta Cantillo, F. 2004 a. Fitocenosis presentes en las áreas costeras del sur de la Sierra Maestra. II. Órdenes Varronio-Phyllostyletalia y Rhytidophyllo-Plumerietalia. Foresta Veracruzana 6: 37-43.

Reyes, O.J. \& Acosta, F. 2004 b. Comunidades secundarias de la Región Oriental de Cuba. IV. Asociaciones Cupanio glabrae - Ocoteetum coriaceae, Ocoteo coriaceae Alvaradoetum arborescentis y Guareo guidoniae Dendropanacetum arborei. Ed. Academia. Biodiversidad de Cuba Oriental. Vol. VII. Pp. 126-139.

Reyes, O.J. \& Acosta, F. 2005. Vegetation. Cuba: National Park La Bayamesa. In: Maceira, D.; Fong, A.; Alverson, W.S. \& Wachter, T. (eds.). Rapid biological inventories: 13. Pp. 113-119 and Appendice 150-161.

Reyes, O.J. \& Martínez, E. 2005. Fitocenosis presentes en las áreas costeras del sur de la Sierra Maestra. IV. Asociaciones Colubrino ellipticae - Gymnanthetum lucidae y Eugenio - Dipholidetum salicifoliae. Foresta Veracruzana 7: 47-52.

Samek, V. 1969. La vegetación de la Isla de Pinos. Academia de Ciencias de Cuba, La Habana. Serie Isla de Pinos 28. 28p.

Samek, V. 1973. Pinares de la Sierra de Nipe; Estudio Sinecológico. Academia de Ciencias de Cuba, La Habana. Serie Forestal, 14. 58p. 
Scamoni, A. \& Passarge, H. 1959. Gedanken zu einer natürlichen Ordnung der Waldgesellschaften. Arch. Forsw 8: 382-426.

Scamoni, A. \& Passarge, H. 1963. Einführung in die praktische Vegetationskunde. 2 Aufl. Jena. 236p.
Viña, N. 1991. Carso. En: Atlas de Santiago de Cuba. Academia de Ciencias de Cuba. Mapa 35.

Zanoni, T.; Mejía, M.; Pimentel, J. \& García, R. 1990. La flora y la vegetación de los Haitises, República Dominicana. Moscosoa 6: 46-89.

Artigo recebido em 19/05/2009. Aceito para publicação em 21/05/2010. 